\title{
Stationary states of the two-dimensional nonlinear Schrödinger model with disorder
}

\author{
Gaididei, Yuri Borisovich; Hendriksen, D.; Christiansen, Peter Leth; Rasmussen, Kim
}

Published in:

Physical Review B

Link to article, DOI:

10.1103/PhysRevB.58.3075

Publication date:

1998

Document Version

Publisher's PDF, also known as Version of record

Link back to DTU Orbit

Citation (APA):

Gaididei, Y. B., Hendriksen, D., Christiansen, P. L., \& Rasmussen, K. (1998). Stationary states of the twodimensional nonlinear Schrödinger model with disorder. Physical Review B, 58(6), 3075-3081.

https://doi.org/10.1103/PhysRevB.58.3075

\section{General rights}

Copyright and moral rights for the publications made accessible in the public portal are retained by the authors and/or other copyright owners and it is a condition of accessing publications that users recognise and abide by the legal requirements associated with these rights.

- Users may download and print one copy of any publication from the public portal for the purpose of private study or research.

- You may not further distribute the material or use it for any profit-making activity or commercial gain

- You may freely distribute the URL identifying the publication in the public portal 


\title{
Stationary states of the two-dimensional nonlinear Schrödinger model with disorder
}

\author{
Yu. B. Gaididei,* D. Hendriksen, and P. L. Christiansen \\ Department of Mathematical Modelling, The Technical University of Denmark, DK-2800 Lyngby, Denmark \\ K. Ф. Rasmussen \\ Theoretical Division, Los Alamos National Laboratory, Los Alamos, New Mexico 87545
}

(Received 11 March 1998)

\begin{abstract}
Solitonlike excitations in the presence of disorder in the two-dimensional cubic nonlinear Schrödinger equation are analyzed. The continuum as well as the discrete problem are analyzed. In the continuum model, otherwise unstable excitations are stabilized in the presence of disorder. In the discrete model, the disorder is found to leave the narrow excitations unaffected. Our results suggest that the disorder provides a possibility to control the spatial extent of the stable excitations in the continuum system. [S0163-1829(98)06429-7]
\end{abstract}

\section{INTRODUCTION}

The interplay between disorder and nonlinearity has been drawing increasing attention of theoretical physicists the last ten years. ${ }^{1}$ It is also a topic of great experimental concern in several fields of modern physics, such as nonlinear optics, ${ }^{2,3}$ polaron formation in solid-states materials, ${ }^{4,5}$ and energy transport in organic thin films. ${ }^{6}$

Attention has mainly been paid to systems that are integrable - soliton bearing - in the absence of disorder. In particular, in Ref. 7 the authors have discussed the effects of a periodic potential on the soliton of the cubic nonlinear Schrödinger (NLS) equation, and have shown on the basis of an averaged NLS equation that the periodic potential leads to a simple renormalization of the solitons and creates a "dressing" of the soliton. Investigations of stationary wave propagation in nonlinear disordered media have shown that nonlinearity changes the transmission properties of disordered systems. ${ }^{8}$ It has been shown theoretically, ${ }^{9}$ and recently verified experimentally ${ }^{10}$ that the presence of nonlinearity may change the characteristic exponential decay of the transmission coefficient with system length into a power-law decay. Among the soliton bearing models is also the so-called selftrapping model of electrons in ionic crystals through polaronic lattice distortion ${ }^{11}$ (a general model for coupled-field systems ${ }^{12}$ ) where the nonlinearity arises from adiabatic elimination of the lattice distortions. The study of such models with disorder and temporal noise has shown that the ground state is always localized in the presence of disorder ${ }^{11}$ while the temporal noise always leads to destruction of the localized states. ${ }^{13}$

However, the studied models all have long-lived solitonlike solutions because in the continuum limit these equations are all exactly integrable. The situation may change drastically if the continuum limit is nonintegrable. A relevant example of such an equation is the two-dimensional (or higherdimensional) NLS equation. The most prominent application of the two-dimensional NLS equations is probably the description of optical beams propagating in a three-dimensional Kerr active medium. ${ }^{14}$ The discrete version has also been applied in the context of optics as a model of optical pulse propagation along a cluster of optical fibers. ${ }^{15}$ Twodimensional NLS equations have also been used in the modeling of two-dimensional organic thin films ${ }^{6}$ and in several other areas of nonlinear physics. However, the importance of the two-dimensional NLS equation stems not only from its physical importance but also from its simple and tractable, yet rich, mathematical structure. As a mathematical object, the two-dimensional NLS equation is rather well studied, mainly due to the critical collapse phenomenon it exhibits (for a review, see Rasmussen and Rypda ${ }^{16}$ ). As was realized early ${ }^{17}$ the two-dimensional NLS equation, although it is nonintegrable, possesses a localized solution. This solution is marginally stable and will, in the presence of perturbations, usually either collapses or disperses.

Recently, Christiansen et al. investigated the effects of disorder on the localized excitations in the quintic onedimensional NLS equation (where the nonlinear term is of the form $|\psi|^{4} \psi$ ) and showed that otherwise unstable excitations are stabilized by the presence of disorder in the continuum problem. ${ }^{18}$ In the present paper, we study the effects of disorder on the localized excitations in the cubic twodimensional NLS equation.

The paper is organized as follows. In Sec. II we introduce the model describing the basic properties in the homogeneous discrete and continuum cases, and we discuss the numerical results obtained when disorder is included in the problem. We present numerical results showing that only the broad excitations are significantly affected by the disorder while the intrinsically localized excitations are rather unaffected. Most importantly, we find that the disorder stabilizes the very broad excitations. In Sec. III we address the problem analytically in the continuum limit and show that the disorder indeed creates a stability window for the localized excitations. Finally, Sec. IV contains a discussion of our results.

\section{MODEL AND NUMERICAL RESULTS}

We consider a quadratic two-dimensional lattice with the lattice spacing equal to unity. The model is given by the Lagrangian

$$
L=i \sum_{\vec{n}} \frac{1}{2}\left(\dot{\psi}_{n} \psi_{n}-\text { c.c. }\right)-H
$$




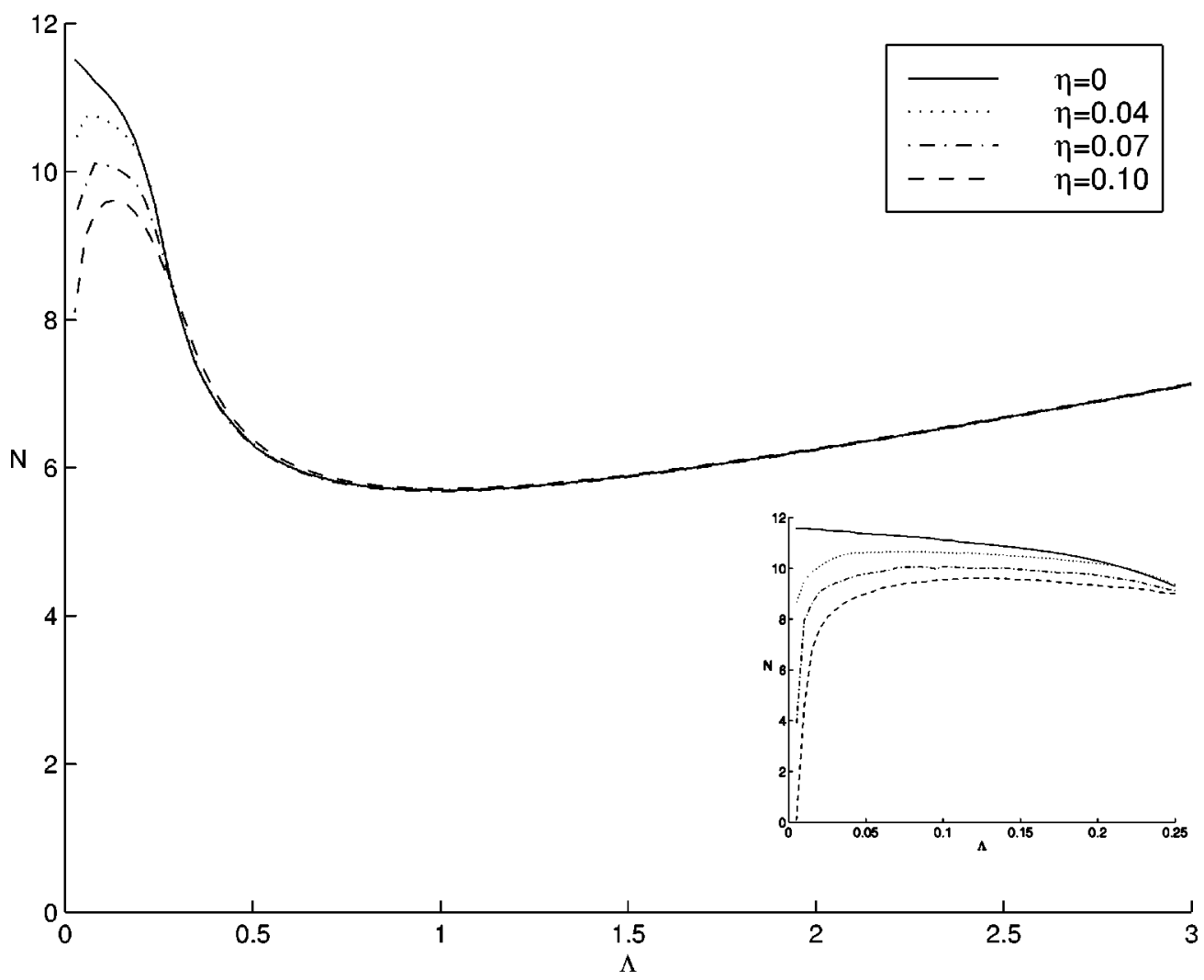

FIG. 1. The norm, $N$ versus nonlinear frequency $\Lambda$ for various disorder strengths $\eta$. Homogeneous case $\eta=0$ (solid line), $\eta=0.04$ (dotted line), $\eta=0.07$ (dashed-dotted line), and $\eta=0.1$ (dashed line).

$$
H=\sum_{\vec{n}} \sum_{\vec{\Delta}}\left|\psi_{n}^{\vec{n}}-\psi_{n}^{\vec{n}-\vec{\Delta}}\right|^{2}-\frac{1}{2} \sum_{\vec{n}}\left|\psi_{n}^{\vec{n}}\right|^{4}-\sum_{\vec{n}} \epsilon_{\vec{n}}^{\vec{n}}\left|\psi_{\vec{n}}^{\vec{n}}\right|^{2}
$$

is the Hamiltonian of the system. In Eqs. (1) and (2), $\psi_{n}$ $\equiv \psi_{m, n}$ is a complex wave function, $\vec{n}=(m, n),(m, n=0$, $\pm 1, \pm 2 \ldots)$ is the lattice vector, while the vector $\vec{\Delta}=$ $( \pm 1,0),(0, \pm 1)$ connects nearest neighbors in the lattice. The first term in Eq. (2) is the dispersive energy of the excitation, the second term describes a self-interaction of the excitation, and the third term represents diagonal disorder in the lattice. Finally, the random functions $\epsilon_{n}^{\vec{n}}$ are assumed to have Gaussian distribution with the probability

$$
p\left(\epsilon_{\vec{n}}^{\vec{*}}\right)=\frac{1}{\eta \sqrt{\pi}} \exp \left[-\left(\epsilon_{n}^{\vec{n}} / \eta\right)^{2}\right]
$$

and have the autocorrelation function

$$
\left\langle\epsilon_{\vec{n}}^{\vec{n}} \epsilon_{n^{\prime}}\right\rangle=\eta^{2} \delta_{\vec{n}, \vec{n}^{\prime}},
$$

where the brackets $\langle\cdots\rangle$ denote averaging over all realizations of the disorder. From the Lagrangian (1) we obtain the equation of motion for the excitation function in the form

$$
\begin{gathered}
i \dot{\psi}_{m, n}+\left(\psi_{m, n-1}+\psi_{m, n+1}+\psi_{m+1, n}+\psi_{m-1, n}-4 \psi_{m, n}\right) \\
+\left|\psi_{m, n}\right|^{2} \psi_{m, n}+\boldsymbol{\epsilon}_{m, n} \psi_{m, n}=0, \\
n, m=0, \pm 1, \pm 2, \ldots
\end{gathered}
$$

Equation (5) conserves the norm $N$ defined as

$$
N=\sum_{\vec{n}}\left|\psi_{\vec{n}}\right|^{2},
$$

and the Hamiltonian $H$.

We are interested in the stationary solutions of Eq. (5) in the form

$$
\psi_{n}^{\vec{n}}(t)=\phi_{\vec{n}} \exp (i \Lambda t),
$$

with a real shape function $\phi_{\vec{n}}$ and a nonlinear frequency $\Lambda$. The governing equations for the functions $\phi_{n} \equiv \phi_{m, n}$ then become

$$
\begin{aligned}
& -\Lambda \phi_{m, n}+\left(\phi_{m, n-1}+\phi_{m, n+1}+\phi_{m+1, n}+\phi_{m-1, n}-4 \phi_{m, n}\right) \\
& +\left|\phi_{m, n}\right|^{2} \phi_{m, n}+\epsilon_{m, n} \phi_{m, n}=0 .
\end{aligned}
$$

Equation (8) together with Eq. (6) constitute a nonlinear eigenvalue problem that can be solved numerically using the techniques described in Ref. 19. The dependence $N(\Lambda)$ in the case without disorder has been studied earlier ${ }^{19-21}$ and is shown with a solid line in Fig. 1. It has previously been shown ${ }^{20-22}$ that the linear stability of the stationary states in the discrete case is determined by the Vakhitov-Kolokolov criterion, ${ }^{23}$ yielding linear stability whenever $d N / d \Lambda>0$. This, together with the solid curve in Fig. 1, shows that lowfrequency nonlinear excitations in the discrete twodimensional NLS model $\left(0 \leqslant \Lambda \leqslant \Lambda_{t h}=1.088\right)$ are unstable. In the continuum limit $(\Lambda \rightarrow 0) N(\Lambda) \rightarrow N_{c} \simeq 11.7$.

Further, we show in Fig. 1 the dependence $N$ on $\Lambda$ for the solutions of Eq. (8) in the presence of disorder. Results for three values of the variance $\eta=0.04$ (dotted line), 0.07 (dash-dotted line), and 0.1 (dashed line) are shown. The re- 

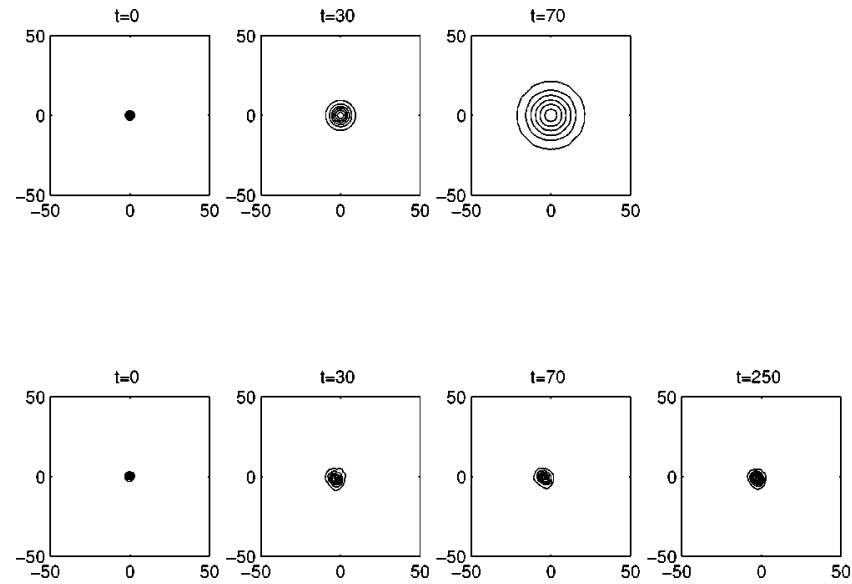

FIG. 2. Evolution of an initial excitation of the norm $N$ $=10.4402$ in continuum systems without disorder (upper part) and with disorder strength $\eta=0.1$ (lower part).

sults have been obtained as averages of 150 realizations of the disorder. Several features arise as a consequence of the disorder. In the continuum limit $(\Lambda \rightarrow 0)$, we no longer have $N=N_{c}$ and $d N / d \Lambda=0$. Instead, $N \rightarrow 0$ and $d N / d \Lambda>0$, signifying that the disorder stabilizes the excitations in the lowfrequency limit. The disorder creates a stability window such that a bistability phenomenon emerges. Consequently, there is an interval of the excitation norm in which two stable excitations with significantly different widths have the same norm.

Furthermore, we see that the disorder creates a gap at small $\Lambda$ in which no localized excitation can exist, and that the size of this gap apparently is increased as the variance of the disorder is increased. It is also clearly seen that, as $\Lambda$ increases (decreasing width), the effect of the disorder vanishes, so that the very narrow excitations are in average unaffected by the disorder and only the continuum results are affected by disorder. It is important to stress that this is an average effect, because for each realization of the disorder the narrow excitation will be affected. The narrow excitation will experience a shift in the nonlinear frequency equal to the amplitude of the disorder at the position of the excitation.

The qualitative form of the dependence $N(\Lambda)$ for a particular realization is very similar to the form of the average dependence shown in Fig. 1. It is noteworthy that for all realizations the curve $N(\Lambda)$ is a smooth curve. It turns out that the basic difference from realization to realization is a displacement of the curve along the $\Lambda$ axis.

The bistability we observe in Fig. 1 occurs due to the competition between two different length scales of the problem, one length scale being defined by the relation between the nonlinearity and the dispersion, while the length scale defined by the disorder gives the other length scale. A similar effect was observed by Christiansen et al. ${ }^{18}$ for the onedimensional discrete NLS equation with a quintic nonlinearity. The latter is quite natural because, as it is well known (see, e.g., Ref. 24), the properties of the two-dimensional NLS model with a cubic nonlinearity are similar to the properties the one-dimensional NLS equation with a quintic nonlinearity.

Having studied the stationary problem, it is vital to compare the results to full dynamical simulations. Therefore, we carry out a numerical experiment launching a pulse in a system governed by the equation (a)

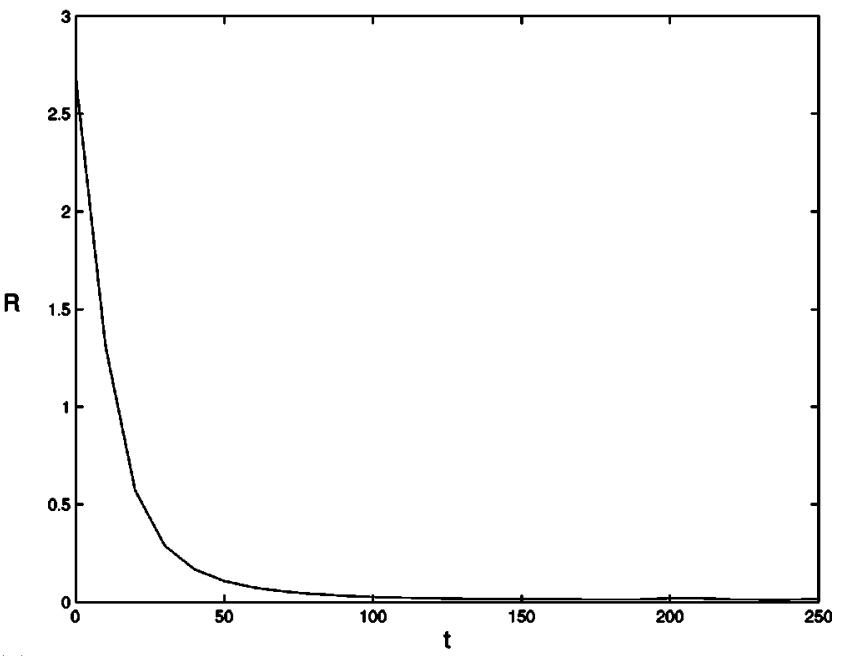

(b)

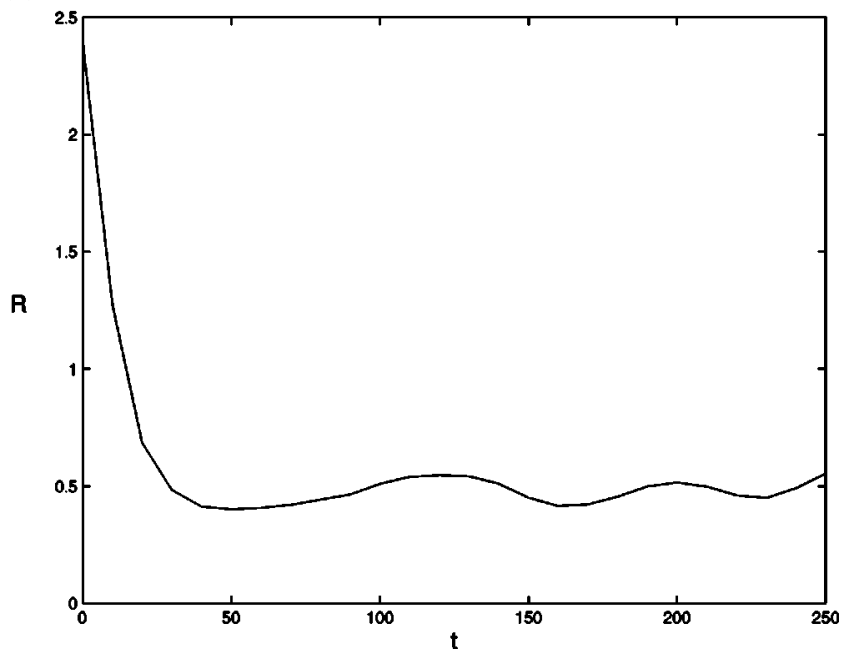

FIG. 3. The inverse width squared, obtained by numerical solution of Eq. (9) in a no-disorder case $(\eta=0)$ (upper part) and with disorder strength $\eta=0.1$ (lower part). The norm $N=10.4402$ is the same as in Fig. 2.

$$
i \partial_{t} \psi(\vec{r}, t)+\nabla^{2} \psi(\vec{r}, t)+|\psi(\vec{r}, t)|^{2} \psi(\vec{r}, t)+\epsilon(\vec{r}) \psi(\vec{r}, t)=0,
$$

being the continuum limit of Eq. (5) for the excitation function $\psi_{n}(t)=\psi(\vec{r}, t)$. Specifically, stationary solutions to Eq. (9) were obtained, and after reducing the amplitude of these solutions by $5 \%$ they were used as initial conditions of the dynamical simulations that were performed by applying a Runge-Kutta scheme. Examples of the described experiment are shown in Fig. 2. As is seen, the pulse behavior in the absence of disorder and in the presence of disorder (we present here a realization corresponding to the disorder variance $\eta=0.1)$ differs drastically. While the pulse rapidly disperses in the ideal system (the contour plot for $t=250$ is absent because the pulse width is of the system size), the process is arrested in the disordered system. After some transient behavior the excitation stabilizes and attains an approximately stationary width. It is clearly seen in Fig. 3 where the quantity

$$
R=\int|\psi(\vec{r}, t)|^{4} d \vec{r}
$$


characterizing the spatial extent of the excitation is shown. When $\psi(\vec{r}, t)$ is chosen as the self-similar solution [see below, Eq. (12)], this quantity represents the squared inverse excitation width. The dynamical simulations thus support the conclusion that otherwise unstable excitations are stabilized by the presence of disorder in the continuum limit.

\section{ANALYTICAL RESULTS}

Apparently, for the average dynamics there are only significant effects of the disorder in the limit of small nonlinear frequencies $\Lambda$, and we shall therefore apply the analytical approach in the continuum limit of Eq. (5) that is given by Eq. (9). In what follows we will not restrict ourselves by the two-dimensional model but will consider the $d$-dimensional $(d=1,2, \ldots)$ case. Therefore, $\vec{r}$ is the $d$-dimensional radius vector and $\nabla^{2}$ is the $d$-dimensional Laplace operator. The potential $\epsilon(\vec{r})$ is a spatially homogeneous Gaussian random function with the properties

$$
\langle\epsilon(\vec{r})\rangle=0, \quad\left\langle\epsilon(\vec{r}) \epsilon\left(\vec{r}^{\prime}\right)\right\rangle=\eta^{2} \delta\left(\vec{r}-\vec{r}^{\prime}\right) .
$$

In order to gain some analytical understanding of how the presence of disorder affects the dynamics of the nonlinear excitations, we apply a collective coordinate approach. Thus it is assumed that the dynamics can be described in terms of collective coordinates using a localized self-similar trial function $\psi(\vec{r}, t)$ (Ref. 25) of the form

$$
\psi(\vec{r}, t)=\sqrt{\frac{A(t)}{b^{d}(t)}} f\left(\frac{|\vec{r}-\vec{R}(t)|}{b(t)}\right) e^{i \alpha(t)[\vec{r}-\vec{R}(t)]^{2}+i \vec{k}(t) \vec{r}+i \phi(t)}
$$

where $f(|\vec{\rho}|)$ is the real shape function. Here we have assumed that the solution is radially symmetric. The two real time-dependent parameters $A(t)$ and $\phi(t)$ determine the amplitude and the phase of the excitation, $b(t)$ and $\alpha(t)$ determine the width and the chirp of the excitation while another pair of real parameters $\vec{R}(t)$ and $\vec{k}(t)$ determine the excitation center-of-mass motion.

Equation (9) is the Euler-Lagrange equation for the action

$$
S=\int_{-\infty}^{\infty} L d t
$$

where

$$
L=\int\left(\frac{i}{2}\left(\psi \partial_{t} \psi^{*}-\text { c.c. }\right)-|\nabla \psi|^{2}+\frac{1}{2}|\psi|^{4}+\epsilon(\vec{r})|\psi|^{2}\right) d \vec{r}
$$

is the Lagrangian of the system. Inserting the trial function into Eq. (9), the following equations are derived from the Euler-Lagrange equations:

$$
\dot{A}=0,
$$

$$
\begin{aligned}
& -A\left(\dot{\phi}+\frac{1}{2} \ddot{\vec{R}} \vec{R}+\frac{1}{4} \dot{\vec{R}}^{2}\right) s(0,2)-\frac{A}{4} \ddot{b} b s(2,2)+\frac{1}{2} \frac{A^{2}}{b^{d}} s(0,4) \\
& \quad=U(b)-F(\{\epsilon\}, b, \vec{R}),
\end{aligned}
$$

$$
\frac{A s(2,2)}{2} \ddot{b}=-\frac{\partial}{\partial b}[U(b)-F(\{\epsilon\}, b, \vec{R})],
$$

and

$$
\frac{1}{2} \ddot{\vec{R}}=\frac{\partial F(\{\epsilon\}, b, \vec{R})}{\partial \vec{R}}
$$

where

$$
U(b)=p(0,1) \frac{A}{b^{2}}-\frac{1}{2} \frac{A^{2}}{b^{d}} s(0,4)
$$

is the effective potential function when disorder is absent and

$$
F(\{\epsilon\}, b, \vec{R})=\frac{A}{b^{d}} \int \epsilon(\vec{r}) f^{2}\left(\frac{|\vec{r}-\vec{R}|}{b}\right) d \vec{r}
$$

is the part of the potential caused by the disorder. The coefficients $s(n, m)$ and $p(n, m)$ are given by

$$
\begin{gathered}
s(n, m)=\int|\vec{r}|^{n} f^{m}(|\vec{r}|) d \vec{r} \\
p(n, m)=\int|\vec{r}|^{n}\left(\frac{d^{m} f(|\vec{r}|)}{d|\vec{r}|^{m}}\right)^{2} d \vec{r} .
\end{gathered}
$$

The stationary points of the set of equations (17) and (18) are determined by the equations

$$
\begin{gathered}
\frac{\partial}{\partial b}[U(b)-F(\{\epsilon\}, b, \vec{R})]=0 \\
\frac{\partial}{\partial \vec{R}} F(\{\epsilon\}, b, \vec{R})=0
\end{gathered}
$$

Solving the problem includes the following steps. Considering the center-of-mass motion that is described by Eq. (18), we see that for each realization of the random potential $\epsilon(\vec{r})$ the pulse moves to the position $\vec{R}=\vec{R}_{m}(\{\epsilon\}, b)$ in the lattice where $F(\{\epsilon\}, b, \vec{R})$ as a function of $\vec{R}$ has a maximum. Inserting the value $\vec{R}=\vec{R}_{m}(\{\epsilon\}, b)$ into Eq. (22),

$$
\frac{\partial}{\partial b} U(b)-\left(\frac{\partial}{\partial b} F(\{\epsilon\}, b, \vec{R})\right)_{\vec{R}=\vec{R}_{m}(\{\epsilon\}, b)}=0
$$

and solving it, we find the value $b(\{\epsilon\})$ of the pulse width that minimizes the potential $U(b)-F(\{\epsilon\}, b, \vec{R})$ for a given realization $\{\epsilon(\vec{r})\}$. The final step of the procedure is to find the average value $\langle b(\{\epsilon\})\rangle$. It is impossible, however, to realize the described program simply because we cannot solve Eqs. (22) and (23), for given $\epsilon(\vec{r})$. Therefore, we will use the following approximate approach.

Introducing

$$
b=B+\delta, \quad B=\langle b\rangle,
$$

and averaging Eq. (24), we get to the zeroth order in $\delta$ that the stationary value of the mean excitation width $B$ is determined by the extrema of the function 


$$
W=U(B)-V
$$

where

$$
V=\left\langle F\left[\{\epsilon\}, \vec{R}_{m}(\{\epsilon\}, B), B\right]\right\rangle
$$

is the averaged disorder potential. Clearly, Eq. (27) can be written as

$$
\left\langle F\left[\{\epsilon\}, \vec{R}_{m}(\{\epsilon\}, B), B\right]\right\rangle=\int_{-\infty}^{\infty} f P(f) d f,
$$

where $P(f) d f$ is the probability of the function $F(\{\epsilon\}, \vec{R}, B)$, having a maximum in the interval $(f, f+d f)$. We also take into account that the random field $F(\{\epsilon\}, \vec{R}, B)$ is stationary (all its spectral moments are independent of $\vec{R}$ ).

To evaluate $V$, we apply the theorem by Belyayev, ${ }^{26}$ which is a multidimensional generalization of Rice's theorem $^{27}$ (see also Ref. 28). Let $F(\vec{R}),\left(\vec{R}=R_{1}, \ldots R_{d}\right)$ be a stationary Gaussian field with zero mean and $F_{i}(\vec{R})$ $=\partial F(\vec{R}) / \partial R_{i}, F_{i j}(\vec{R})=\partial F(\vec{R}) / \partial R_{i} \partial R_{j}$. Let $\mathcal{D}=\left(h_{i j}\right)$ be a symmetric $d \times d$ matrix and $\vec{g}=\left(g_{i}\right)$ a vector of length $d$, and $\vec{h}=\left(h_{i j}\right),(i \leqslant j=1,2 . . d)$ a vector of length $d(d+1) / 2$. Finally, if $p(f, \vec{g}, \vec{h})$ is the joint probability density function for the random functions $F(\vec{R}), F_{i}(\vec{R}), F_{i j}(\vec{R})$, so that

$$
p(f, \vec{g}, \vec{h})=\left\langle\delta(f-F) \prod_{i=1}^{d} \delta\left(g_{i}-F_{i}\right) \prod_{i \leqslant j=1}^{d(d+1) / 2} \delta\left(h_{i j}-F_{i j}\right)\right\rangle .
$$

The probability that $F$ has a maximum in the interval $(f, f$ $+d f)$ is

$$
P(f) d f=(-1)^{d} \frac{\int_{\mathcal{P}(h)}|\mathcal{D}(h)| p(f, 0, \vec{h}) d \vec{h}}{\int_{-\infty}^{\infty} \int_{\mathcal{P}(h)}|\mathcal{D}(h)| p(f, 0, \vec{h}) d \vec{h} d f} d f,
$$

where $|\mathcal{D}(h)|$ is the determinant of $\mathcal{D}(h)$ and $\mathcal{P}(h)$ is the set of $\vec{h}$ for which $\mathcal{D}(h)$ is negative definite.

Inserting Eq. (30) into Eq. (28), we get

$$
\begin{aligned}
&\left\langle F\left(\{\epsilon\}, \vec{R}_{m}(\{\epsilon\}, B), B\right)\right\rangle \\
&=(-1)^{d} \frac{\int_{-\infty \mathcal{P}(h)}^{\infty} f|\mathcal{D}(h)| p(f, 0, \vec{h}) d \vec{h} d f}{\int_{-\infty}^{\infty} \int_{\mathcal{P}(h)}|\mathcal{D}(h)| p(f, 0, \vec{h}) d \vec{h} d f} .
\end{aligned}
$$

It is seen from Eqs. (11) and (20) that (i) $F$ and $F_{i}$ are uncorrelated for all $i$, (ii) $F$ and $F_{i j}$ are uncorrelated for $i$ $\neq j$, (iii) $F_{i}$ and $F_{j}$ are uncorrelated for $i \neq j$, (iv) $F_{i}$ and $F_{j k}$ are uncorrelated for all $i, j, k$.

Thus we obtain from Eqs. (11), (20), (29), and (31),

$$
\left\langle F\left(\{\epsilon\}, \vec{R}_{m}(\{\epsilon\}, B), B\right)\right\rangle
$$

$$
=(-1)^{d} \frac{\int_{\mathcal{P}(h)}|\mathcal{D}(h)| \sum_{j=1}^{d} M_{j} \frac{\partial}{\partial h_{j j}} \phi(\vec{h}) d \vec{h}}{\int_{\mathcal{P}(h)}|\mathcal{D}(h)| \phi(\vec{h}) d \vec{h}},
$$

where the abbreviation

$$
\phi(\vec{h})=\exp \left(-\frac{1}{2} \sum_{i<j=1}^{d} \frac{h_{i j}^{2}}{M_{i j}}-\frac{1}{2} \sum_{i, j=1}^{d} h_{i i} h_{j j}\left[M^{-1}\right]_{i j}\right)
$$

is used. Here

$$
M_{j}=-\left\langle F F_{j j}\right\rangle=\left\langle F_{j} F_{j}\right\rangle,
$$

and the square $d \times d$ matrix $M$ with components $M_{i j}$ that are second moments of the $F_{i j}$ 's, i.e.,

$$
M_{i j}=\left\langle F_{i i} F_{j j}\right\rangle \text {. }
$$

An explicit form of the effective potential function $W$ is obtained using the fact that the trial function given by Eq. (12) is radially symmetric in the frame of reference that is coupled with the center-of-mass $R$. Then we have

$$
\begin{aligned}
M_{j} & =\frac{A^{2} \eta^{2}}{B^{2 d}} \int\left(\frac{\partial g\left(\frac{|\vec{r}|}{B}\right)}{\partial r_{j}}\right){ }^{2} d \vec{r} \\
& =\frac{A^{2} \eta^{2}}{B^{d+2}} \int \frac{r_{j}^{2}}{r^{2}}\left[g^{\prime}(|\vec{r}|)\right]^{2} d \vec{r} \equiv \frac{A^{2} \eta^{2}}{B^{d+2}} m_{1},
\end{aligned}
$$

$$
\begin{aligned}
M_{j j}= & \frac{A^{2} \eta^{2}}{B^{2 d}} \int\left(\frac{\partial^{2} g\left(\frac{|\vec{r}|}{B}\right)}{\partial r_{j}^{2}}\right){ }^{2} d \vec{r} \\
= & \frac{A^{2} \eta^{2}}{B^{d+4}} \int\left[\frac{1}{|\vec{r}|}\left(1-\frac{r_{j}^{2}}{|\vec{r}|^{2}}\right) g^{\prime}(|\vec{r}|)+\frac{r_{j}^{2}}{|\vec{r}|^{2}} g^{\prime \prime}(|\vec{r}|)\right]^{2} d \vec{r} \\
\equiv & \frac{A^{2} \eta^{2}}{B^{d+4}} m_{2}, \\
M_{i j} & \left.=\frac{A^{2} \eta^{2}}{B^{2 d}} \int \frac{\left.\partial^{2} g\left(\frac{|\vec{r}|}{B}\right)\right)^{2}}{\partial r_{i} \partial r_{j}}\right)^{d \vec{r}} \\
& =\frac{A^{2} \eta^{2}}{B^{d+4}} \int \frac{r_{i}^{2} r_{j}^{2}}{|\vec{r}|^{4}}\left(g^{\prime \prime}(|\vec{r}|)-\frac{1}{|\vec{r}|} g^{\prime}(|\vec{r}|)\right)^{2} d \vec{r} \\
& \equiv \frac{A^{2} \eta^{2}}{B^{d+4}} m_{3}, \quad(i \neq j),
\end{aligned}
$$

where $g=f^{2}$ and $g^{\prime}(x)=d g / d x$. It is seen that the coefficients $m_{1}, m_{2}$, and $m_{3}$ do not depend on the indices $i$ and $j$. They merely depend on the dimensionality of the system and on the explicit form of the trial function $f(r)$.

Inserting Eqs. (36) into Eqs. (32)-(35), and rescaling the variables $h_{i j}$, 


$$
h_{i j}=\frac{A \eta}{B^{d / 2+2}} z_{i j}
$$

we obtain that the averaged disorder potential has the form

$$
V=v_{d} \frac{A \eta}{B^{d / 2}}
$$

where the coefficient $v$ is

$$
v_{d}=(-1)^{d} m_{1} \frac{\int_{\mathcal{P}(z)}|\mathcal{D}(z)| \sum_{j=1}^{d} \frac{\partial}{\partial z_{j j}} \phi(\vec{z}) d \vec{z}}{\int_{\mathcal{P}(z)}|\mathcal{D}(z)| \phi(\vec{z}) d \vec{z}}
$$

with the function $\phi(\vec{z})$ being determined by Eqs. (33) and (35) in which one has to substitute

$$
M_{i j}=m_{2} \delta_{i j}+m_{3}\left(1-\delta_{i j}\right) .
$$

In accordance with Eqs. (36), the coefficient $v$ depends only on the dimensionality of the system $d$ and the explicit form of the trial function $f(r)$. E.g.,

$$
v_{1}=\sqrt{2 \pi} \frac{p(0,1)}{\sqrt{p(0,2)}}, \quad v_{2}=\frac{8}{\sqrt{6 \pi}} \frac{p(0,1)}{\sqrt{p(-2,1)+p(0,2)}}
$$

Thus the effective averaged potential $W$ takes the form

$$
W=p(0,1) \frac{A}{B^{2}}-\frac{1}{2} \frac{A^{2}}{B^{d}} s(0,4)-v_{d} \frac{A \eta}{B^{d / 2}} .
$$

Using this and Eq. (16), the nonlinear frequency $\dot{\phi}=\Lambda$ can be determined as

$$
\Lambda=-\frac{1}{N}\left(W-\frac{1}{2} \frac{A^{2} s(0,4)}{B^{d}}\right)
$$

In the case of the one-dimensional NLS equation ( $d$ =1), Eq. (42) has the same form as the effective potential obtained in Ref. 11 where the effects of disorder on the polaron ground state were studied. In Ref. 11 a quite different approach, combining statistical and scaling analysis, was used.

In the case of the two-dimensional NLS equation ( $d$ $=2$ ), the effective potential (42) can be written as

$$
W=\frac{N}{s(0,2)}\left[p(0,1)\left(1-\frac{N}{N_{c}}\right) \frac{1}{B^{2}}-\frac{v_{2} \eta}{B}\right],
$$

where $N=A s(0,2)$ is the number of excitations and $N_{c}$ $=2 p(0,1) s(0,2) / s(0,4)$ is its critical value for which the stationary state of the two-dimensional NLS equation exists in the absence of disorder. When $N<N_{c}$, the effective potential (44) has a minimum for

$$
B=\frac{2}{v_{2} \eta} p(0,1)\left(1-\frac{N}{N_{c}}\right)
$$

and the nonlinear frequency (43) takes the form

$$
\Lambda=\frac{v_{2}^{2} \eta^{2}}{4 s(0,2) p(0,1)\left(1-\frac{N}{N_{c}}\right)}\left(1+\frac{A s(0,4)}{2 p(0,1)\left(1-\frac{N}{N_{c}}\right)}\right) .
$$

Equation (46) shows that the nonlinear frequency $\Lambda$ increase monotonically when $N$ is increasing. According to the Vakhitov-Kolokolov criterion, this means that the corresponding stationary states are linearly stable. It is also seen from Eq. (46) that $\Lambda(N)$ does not vanish in the limit $N$ $\rightarrow 0$ :

$$
\Lambda_{\text {gap }} \equiv \lim _{N \rightarrow 0} \Lambda \sim \eta^{2}
$$

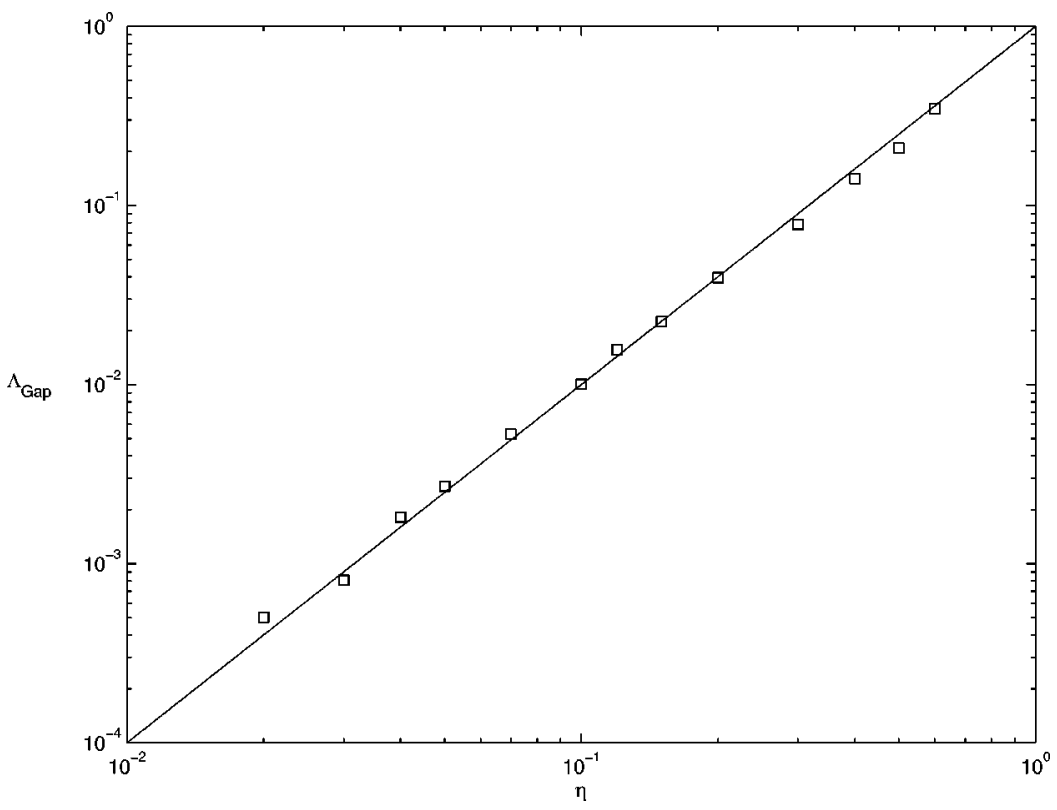

FIG. 4. The width of the gap, $\Lambda_{\text {gap }}$ versus the disorder strength $\eta$. Results of numerical calculations are indicated by squares while the analytical dependence given by Eq. (47) is shown by the solid line. The gap is measured at $N=4$. 
The appearance of the gap was also seen in the numerical simulations. Figure 4 shows the comparison of the dependence given by Eq. (47) with the numerically obtained dependence of the gap. The agreement between the two approaches is rather good, signifying that the analytical approach captures the essential features of the system.

\section{SUMMARY}

In summary, we have in this paper shown that the presence of disorder permits the existence of stable localized low-frequency excitations. We have shown this using analytical analysis and numerical simulations of the stationary as well as the dynamical problem. Analyzing the discrete problem, the appearance of a bistability phenomenon was observed, and the source of this bistability was identified to be the competition between two length scales. The length scale was found to be directly related to the strength of the disorder. The existence of a frequency gap in which no stationary state exists was shown analytically and numerically. This gap, and the appearance of a narrow region where stable excitations exist, allows rather accurate controllability of the excitations via the disorder. Comparing our results with the results of Ref. 18 for a similar case of a one-dimensional NLS equation with a quintic nonlinearity, it is found that the two systems exhibit very similar features in the presence of disorder. This may be predictable considering the similarity of these systems. ${ }^{16}$ It is, however, noteworthy that the averaged potential, Eq. (39), arising from the disorder is different in the two cases [see Eq. (43) of Ref. 18], suggesting that the exact form of the potential is not crucial.

\section{ACKNOWLEDGMENTS}

Yu.B.G. thanks MIDIT and the Department of Mathematical Modelling, Technical University of Denmark for hospitality. He also acknowledges partial support from the Ukrainian Fundamental Research Fund under Grant No. 2.4/ 355 and from SRC QM "Vidhuk." The present work was supported by the Danish Research Council through Contract No. 9313393 (supercomputing projects). Work at Los Alamos National Laboratory was performed under the auspices of the US DOE.
*Permanent address: Bogolyubov Institute for Theoretical Physics, 252143 Kiev, Ukraine.

${ }^{1}$ See, e.g, Disorder and Nonlinearity, edited by A. R. Bishop, D. K. Campbell, and S. Pnevmatikos (Springer-Verlag, Berlin, 1989); Nonlinearity with Disorder, edited by F. K. Abdullaev, A. R. Bishop, S. Pnevmatikos, and E. N. Economou (Springer, Berlin, 1992); Fluctuation Phenomena: Disorder and Nonlinearity, edited by A. R. Bishop, S. Jiménez, and L. Vázquez (World Scientific, Singapore, 1995).

${ }^{2}$ H. Hasegawa and Y. Kodama, Solitons in Optical Communications (Clarendon, Oxford, 1995).

${ }^{3}$ F. Abdullaev, Theory of Solitons in Inhomogeneous Media (Wiley, New York, 1994).

${ }^{4}$ N. Mott, Conduction in Non-Crystalline Materials (Oxford University Press, Oxford, 1987).

${ }^{5}$ Electron-Electron Interaction in Disordered Systems, edited by A. L. Efros and M. Pollak (North-Holland, Amsterdam, 1985).

${ }^{6}$ K. Ø. Rasmussen, Yu. B. Gaididei, O. Bang, and P. L. Christiansen, Math. Comput. Simul. 40, 339 (1996), and references therein.

${ }^{7}$ R. Scharf and A. R. Bishop, Phys. Rev. E 47, 1375 (1993); R. Scharf, Chaos Solitons Fractals 5, 2527 (1995).

${ }^{8}$ P. Devillard and B. Souilard, J. Stat. Phys. 43, 423 (1986); B. Douçot and R. Rammel, Europhys. Lett. 3, 969 (1987).

${ }^{9}$ Yu. S. Kivshar, S. A. Gredeskul, A. Sánchez, and L. Vázquez, Phys. Rev. Lett. 64, 1693 (1990).

${ }^{10}$ V. A. Hopkins, J. Keat, G. D. Meegan, T. Zhang, and J. D. Maynard, Phys. Rev. Lett. 76, 1102 (1996).

${ }^{11}$ M. N. Bussac, G. Mamalis, and P. Mora, Phys. Rev. Lett. 75, 292 (1995).

${ }^{12}$ J. C. Eilbeck, P. S. Lomdahl, and A. C. Scott, Physica D 16, 318 (1985).

${ }^{13}$ P. L. Christiansen, Yu. B. Gaididei, M. Johansson, and K. Ф. Rasmussen, Phys. Rev. B 55, 5759 (1997).
${ }^{14}$ A. C. Newell and J. V. Moloney, Nonlinear Optics (AddisonWiley, Amsterdam, 1992).

${ }^{15}$ A. B. Aceves, G. G. Luther, C. DeAngelis, A. M. Rubenchik, and S. K. Turitsyn, Phys. Rev. Lett. 75, 73 (1995).

${ }^{16}$ J. Juul Rasmussen and K. Rypdal, Phys. Scr. 33, 481 (1986); K. Rypdal and J. Juul Rasmussen, ibid., 33, 498 (1986).

${ }^{17}$ R. I. Chiao, E. Garmire, and C. H. Townes, Phys. Rev. Lett. 13, 479 (1964).

${ }^{18}$ P. L. Christiansen, Yu. B. Gaididei, M. Johansson, K. Ø. Rasmussen, D. Usero, and L. Vázquez, Phys. Rev. B 56, 14407 (1997).

${ }^{19}$ P. L. Christiansen, Yu. B. Gaididei, K. Ф. Rasmussen, V. K. Mezentsev, and J. J. Rasmussen, Phys. Rev. B 54, 900 (1996).

${ }^{20}$ V. K. Mezentsev, S. L. Musher, I. V. Ryzhenkova, and S. K. Turitsyn, Pis'ma Zh. Eksp. Teor. Fiz. 60, 815 (1994) [JETP Lett. 60, 829 (1994)].

${ }^{21}$ E. W. Laedke, K. H. Spatchek, V. K. Mezentsev, S. L. Musher, I. V. Ryzhenkova, and S. K. Turitsyn, Pis'ma Zh. Eksp. Teor. Fiz. 62, 652 (1995) [JETP Lett. 62, 677 (1995)].

${ }^{22}$ E. W. Laedke, K. H. Spatschek, and S. K. Turitsyn, Phys. Rev. Lett. 73, 1055 (1994); E. W. Laedke, O. Kluth, and K. H. Spatschek, Phys. Rev. E 54, 4299 (1996).

${ }^{23}$ M. G. Vakhitov and A. A. Kolokolov, Izv. Vyssh. Uch. Zav. Radiofizika 16, 1020 (1973) [Radiophys. Quantum Electron. 16, 783 (1973)].

${ }^{24}$ O. Bang, J. Juul Rasmussen, and P. L. Christiansen, Nonlinearity 7, 205 (1994).

${ }^{25}$ M. Landman, G. Papanicolau, C. Sulem, and P. Sulem, Physica D 47, 393 (1991).

${ }^{26}$ Yu. K. Belyayev, in Proceedings of the 6th Berkeley Symposium on Mathematical Statistics and Probability, 1970, edited by L. M. Le Cam, J. Neyman, and E. L. Scott (University of California Press, Berkeley, 1972), Vol. III, pp. 1-17.

${ }^{27}$ S. O. Rice, Bell Syst. Tech. J. 23, 282 (1944).

${ }^{28}$ O. Krée and C. Soize, Mathematics of Random Phenomena (Reidel, Dordrecht, 1986). 